\title{
SURFACE SUBGROUPS OF GRAPH GROUPS
}

\author{
HERMAN SERVATIUS, CARL DROMS, AND BRIGITTE SERVATIUS
}

(Communicated by Warren J. Wong)

\begin{abstract}
Given a graph $\Gamma$, define the group $F_{\Gamma}$ to be that generated by the vertices of $\Gamma$, with a defining relation $x y=y x$ for each pair $x, y$ of adjacent vertices of $\Gamma$. In this article, we examine the groups $F_{\Gamma}$, where the graph $\Gamma$ is an $n$-gon, $(n \geq 4)$. We use a covering space argument to prove that in this case, the commutator subgroup $F_{\Gamma}^{\prime}$ contains the fundamental group of the orientable surface of genus $1+(n-4) 2^{n-3}$. We then use this result to classify all finite graphs $\Gamma$ for which $F_{\Gamma}^{\prime}$ is a free group.
\end{abstract}

To each graph $\Gamma=(V, E)$, with vertex set $V$ and edge set $E$, we associate a presentation $P \Gamma$ whose generators are the elements of $V$, and whose relations are $\{x y=y x \mid x, y$ adjacent vertices of $\Gamma\} . P \Gamma$ can be regarded as the presentation of a $k$-algebra $k \Gamma$, of a monoid $M_{\Gamma}$, or of a group $F_{\Gamma}$, called a graph group. These objects have been previously studied by various authors [2-8].

Graph groups constitute a subclass of the Artin groups. Recall that an Artin group is defined by a presentation whose relations all take the form $x y x \cdots=$ $y x y \cdots$, where the two sides have the same length $n>1$, and there is at most one such relation for any pair of generators. To each such presentation we can associate a labeled graph $\Gamma$, which has a vertex for each generator, and for each relation $x y x \cdots=y x y \cdots$, an edge joining $x$ and $y$ and labeled " $n$ ", where $n$ is the length of each side of the relation. Thus, a graph group is an Artin group whose graph has all edges labeled ' 2 '. In the same context, we mention the conjecture of Tits [1], which states that in the Artin group with labeled graph $\Gamma$, the subgroup generated by the squares of the generators is isomorphic to the graph group $F_{\Gamma_{2}}$, where $\Gamma_{2}$ is the subgraph of $\Gamma$ consisting of all the vertices, and all edges labeled ' 2 '. This conjecture has been proved by $S$. Pride [7] in the case that the graph $\Gamma$ contains no triangles.

For many graphs $\Gamma$ it is true that every subgroup of $F_{\Gamma}$ is itself a graph group [4], besides the obvious cases where $\Gamma$ is either complete $\left(F_{\Gamma}\right.$ free Abelian) or

Received by the editors May 23, 1988 and, in revised form, July 15, 1988. This paper was presented at the AMS joint meeting in College Park, Maryland, April 1988.

1980 Mathematics Subject Classification (1985 Revision). Primary 20F05; Secondary 57M05. 
completely disconnected $\left(F_{\Gamma}\right.$ free). In this article, we use topological techniques to examine the commutator subgroups of certain graph groups, showing in particular that some of them are not graph groups.

For $n \geq 3$, the $n$-gon is the graph with $n$ vertices $v_{1}, v_{2}, \ldots, v_{n}$, and $n$ edges $\left(v_{i}, v_{i+1}\right)$, indices modulo $n$. We will show that if $\Gamma$ has a subgraph isomorphic to an $n$-gon for some $n \geq 4$, then the commutator subgroup $F_{\Gamma}^{\prime}$ has a subgroup isomorphic to the fundamental group of the orientable surface of genus $1+(n-4) 2^{n-3}$. (In particular, the graph group of the pentagon contains a subgroup isomorphic to the group of the five-holed torus.) We will use this result to show that $F_{\Gamma}^{\prime}$ is a free group if and only if $\Gamma$ contains no full subgraph isomorphic to any $n$-gon with $n \geq 4$. We also show that if $\Gamma$ does not contain any squares (i.e., full subgraphs isomorphic to $C_{4}$ ), then $F_{\Gamma}^{\prime}$ can be a graph group only if it is free.

\section{Preliminaries}

A graph is a pair $(V, E)$, where $V$ is the set of vertices and $E$ is a set of unordered pairs of elements of $V$. So a graph is undirected with no loops and no multiple edges. A graph $\Sigma=(W, D)$ is called a subgraph of $\Gamma$ if $W \subset V$ and $D \subset E$, and there is a natural homomorphism $f: F_{\Sigma} \rightarrow F_{\Gamma}$ defined by setting $f(w)=w$ for each $w \in W$. If $D$ contains all unordered pairs of elements of $W$ which are contained in $E$, we call $\Sigma$ a full subgraph of $\Gamma$. In this case, it is clear that the natural homomorphism is one-to-one, so we shall simply regard $F_{\Sigma}$ as a subgroup of $F_{\Gamma}$.

We now summarize a result of [8], which completely describes the centralizer of an element of $F_{\Gamma}$. Given an element $u \in F_{\Gamma}$, the support of $u$, $\operatorname{supp}(u)$, is the set of vertices $v \in V$ such that either $v$ or $v^{-1}$ occurs in all factorizations of $u$ as a product of vertices and their inverses. $\operatorname{Supp}(u)$ is well defined.

Let $1 \neq x \in F_{\Gamma}$. Then there are sets $A_{0}, A_{1}, \ldots, A_{n}$ of vertices of $\Gamma$, and a factorization $p^{-1}\left(x_{1}^{r_{1}} \cdots x_{n}^{r_{n}}\right) p$ of $x$ such that (1) for $i=1,2, \ldots, n$, $A_{i}=\operatorname{supp}\left(x_{i}\right),(2)$ the sets $A_{0}, A_{1}, \ldots, A_{n}$ are pairwise disjoint, (3) if $i \neq j$, then every vertex in $A_{i}$ is adjacent to each vertex in $A_{j}$, and (4) $\operatorname{cent}(x)=$ $p^{-1}\left(G \times\left\langle x_{1}\right\rangle \times \cdots \times\left\langle x_{n}\right\rangle\right) p$, where $G$ is the subgroup of $F_{\Gamma}$ generated by the elements of $A_{0}$. The elements $x_{i}$ are called the pure factors of $x$.

\section{TOPOlOgical REALIZATION OF THE COMMUTATOR SUBGROUP}

Let $\Gamma=(V, E)$ be a finite graph, and let $X_{\Gamma}$ denote the Cayley complex of the corresponding presentation of $F_{r}$; that is, $X_{\Gamma}$ has one 0 -cell, *, an oriented 1-cell for each vertex of $\Gamma$, and for each edge $(v, w) \in E$, a 2-cell attached along the loop $v w v^{-1} w^{-1}$. We have $\pi_{1}\left(X_{\Gamma}\right)=F_{\Gamma}$. If $\Gamma=(V, E)$ has $n$ vertices, then $X_{\Gamma}$ is a subcomplex of the $n$-fold Cartesian product $\left(S^{1}\right)^{n}$, where the circle $S^{1}$ has one 0 -cell and $\left(S^{1}\right)^{n}$ is the product complex. In particular, if $K$ is the complete graph with vertex set $V$, then $X_{K}$ is the entire 2-skeleton of 
$\left(S^{1}\right)^{n}$. Let $U_{K}$ denote the universal cover of $X_{K}$. Since the fundamental group of a complex is carried by its 2-skeleton, it follows that $U_{K}$ is the 2-skeleton of the cubical complex of $R^{n}$, i.e., the complex on $R^{n}$ whose $n$-cells are the integer translates of the unit cube $I^{n}$.

Now, it is easy to see that the natural homomorphism $\alpha: F_{\Gamma} \rightarrow F_{K}$ is the Abelianization map, and that the inclusion $i: X_{\Gamma} \rightarrow X_{K}$ realizes $\alpha$. Thus, $F_{\Gamma}^{\prime}=\operatorname{ker}(\alpha)$ is realized by $U G$ in the pullback diagram

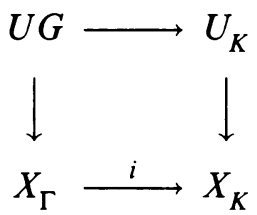

Hence, $U G$ is the subcomplex of $U_{K}$ obtained by deleting the lifts of all 2-cells of $X_{K}$ which correspond to nonadjacent vertices of $\Gamma$.

Let $w$ be a word on $V^{ \pm 1}$ representing an element $[w] \in F_{\Gamma}$. Then [8] $w$ can be transformed into a word of shortest length representing $[w]$ via a finite sequence of the following moves:

$\mathbf{M}_{1}$. Delete a subword $a^{-1} a$ or $a a^{-1}$.

$\mathbf{M}_{2}$. Replace a subword $v^{ \pm 1} w^{ \pm 1}$ with $w^{ \pm 1} v^{ \pm 1}$ if $(v, w) \in E$.

In particular, if $[w]=1$, then $w$ can be transformed into the empty word using a finite sequence of these moves.

Let $Z$ be a covering space of $X_{\Gamma}$, and let $Z$ have the induced cell structure. Let $p: I \rightarrow Z$ be any (cellular) loop in $Z$ which is path-homotopic to the constant loop. Then, by the above, there is a sequence of path homotopies

$$
p=p_{1} \rightarrow p_{2} \rightarrow \cdots \rightarrow p_{k}=*
$$

connecting $p$ to the constant loop $*$, with each $p_{i}$ cellular, such that the homotopy $p_{i} \rightarrow p_{i+1}$ is supported either by $p_{i}$, in case of a move of type $M_{1}$, or, in case of a move of type $M_{2}$, by $p_{i} \cup F$, where $F$ is a face of $Z$ which intersects $p_{i}$ in at least two incident edges (i.e., two edges having a common endpoint). Let $Y$ be a subcomplex of $Z$ and suppose that every face of $Z$ which intersects $Y$ in at least two incident edges is contained in $Y$. Then any loop in $Y$ which is path homotopic to the constant loop in $Z$ is path homotopic to the constant loop in $Y$ also. Thus:

Proposition 1. Let $Z$ be a cover of the Cayley complex of $F_{\Gamma}$, and let $Y$ be a subcomplex of $Z$ with the property that any face of $Z$ which contains at least two incident edges of $Y$ is contained in $Y$. Then the inclusion $i: Y \rightarrow Z$ induces a monomorphism $i_{*}: \pi_{1}(Y) \rightarrow \pi_{1}(Z)$.

\section{SURFACE SUBGROUPS OF $n$-GON GROUPS}

Let $C_{n}$ denote the $n$-gon $(n \geq 3)$, and $F_{n}$ the corresponding graph group. As in $\S 2, F_{n}^{\prime}$ is realized by a subcomplex $U_{n}$ of the cubical lattice of $R^{n}$. 
Consider the subcomplex $I^{n}$ of $R^{n}$. Since $I^{n}$ is convex, every 2-cell of $R^{n}$ which intersects $I^{n}$ in two edges is also a 2-cell of $I^{n}$, and so $Y=U_{n} \cap I^{n}$ has the same property with respect to $U_{n}$. Thus, by Proposition $1, \pi_{1}(Y)$ is a subgroup of $F_{n}^{\prime}$.

Now, every edge of $I^{n}$ corresponds to a vertex $v$ of $C_{n}$, and is incident to $n-1$ faces, one for each of the $n-1$ other vertices of $C_{n}$. $Y$ contains exactly two of these faces-those corresponding to the two vertices of $C_{n}$ which are adjacent to $v$. Thus, $Y$ is a connected 2-complex in which every edge is adjacent to exactly two faces; i.e., $Y$ is a surface. $Y$ is 2 -sided since it is a subcomplex of the 2-skeleton of $I^{n}$.

To compute the genus of $Y$, we observe that $I^{n}$ has $2^{n}$ vertices, $n 2^{n-1}$ edges and $\frac{1}{2} n(n-1) 2^{n-2}$ faces, with $2^{n-2}$ faces for each of the $\frac{1}{2} n(n-1)$ pairs of vertices in $C_{n}$. Since only $n$ of these pairs are adjacent in $C_{n}, Y$ has only $n 2^{n-2}$ faces, so the Euler characteristic of $Y$ is

$$
\chi(Y)=2^{n}-n 2^{n-1}+n 2^{n-2}=(4-n) 2^{n-2}
$$

and the genus of $Y$ is $1-\frac{1}{2} \chi(Y)=1+(n-4) 2^{n-3}$.

Thus, we have shown

Theorem 1. Let $F_{n}$ be the graph group of the n-gon graph. Then $F_{n}^{\prime}$ has a subgroup isomorphic to the fundamental group of the orientable surface of genus $1+(n-4) 2^{n-3}$.

\section{COMMUTATOR SUBGROUPS OF GRAPH GROUPS}

Let $\Gamma=(V, E)$ be a graph. Because the exponent sum of each letter of $V$ in each relator of $F_{\Gamma}$ is 0 , a word $w$ on $V^{ \pm 1}$ represents an element of $F_{\Gamma}^{\prime}$ if and only if the exponent sum on each letter of $V$ in $w$ is 0 . It follows that if $\Sigma$ is a full subgraph of $\Gamma$, then $F_{\Sigma}^{\prime}=F_{\Sigma} \cap F_{\Gamma}^{\prime}$, and that if $x \in F_{\Gamma}^{\prime}$, then each pure factor of $x$ lies in $F_{\Gamma}^{\prime}$.

A graph $\Gamma$ is called triangulated if it contains no full subgraph isomorphic to an $n$-gon for any $n \geq 4$. (In particular note that trees are triangulated.)

Theorem 2. If $\Gamma$ is finite, then $F_{\Gamma}^{\prime}$ is free if and only if $\Gamma$ is triangulated.

Proof. If $\Gamma$ contains a full $n$-gon with $n \geq 4$, then $F_{\Gamma}^{\prime}$ contains the group of some surface of positive genus, and so cannot be free. Conversely, suppose $\Gamma$ is triangulated. If $\Gamma$ is complete, $F_{\Gamma}^{\prime}$ is trivial. Otherwise [4], $\Gamma$ can be written as the union of two subgraphs $X$ and $Y$ whose intersection, $K$, is a complete graph (empty in case $\Gamma$ is disconnected). Comparing presentations, we find that $F_{\Gamma}=F_{X}{ }^{*} F_{K} F_{Y}$. Now, $F_{\Gamma}^{\prime} \cap F_{X}=F_{X}^{\prime}, F_{\Gamma}^{\prime} \cap F_{Y}=F_{Y}^{\prime}$, and $F_{\Gamma}^{\prime} \cap F_{K}=F_{K}^{\prime}=\{1\}$ since $F_{K}$ is Abelian. But $X$ and $Y$ are full subgraphs of $\Gamma$, so they are both triangulated, and so by induction, $F_{X}^{\prime}$ and $F_{Y}^{\prime}$ are free. Therefore, $F_{\Gamma}^{\prime}$ is free, by the Kurosh subgroup theorem. 
Lemma 1. Let $\Gamma$ be a graph which contains no full squares (i.e., no full subgraph isomorphic to the square), and let $x \in F_{\Gamma}^{\prime}, x \neq 1$. Then cent $(x)$, the centralizer of $x$ in $F_{\Gamma}$, is free Abelian, and cent $\left(F_{\Gamma}^{\prime} ; x\right)$, the centralizer of $x$ in $F_{\Gamma}^{\prime}$, is cyclic. Proof. Write $x=p^{-1}\left(x_{1}^{r_{1}} \cdots x_{n}^{r_{n}}\right) p$, and let $A_{0}, A_{1}, \ldots, A_{n}$ and $G$ be as in paragraph 1. Since $x \neq 1$, one of the $x_{i}$, say $x_{1}$, must be nontrivial, and since $x_{1} \in F_{\Gamma}, A_{1}$ must contain two nonadjacent vertices. But then each of the sets $A_{0}, A_{1}, \ldots, A_{n}$ must induce a complete subgraph of $\Gamma$, since $\Gamma$ contains no squares (for recall that each vertex in any of these sets is adjacent to every vertex of $A_{1}$ ). In particular, $G$ is free Abelian. Further, the support of a nontrivial element of $F_{r}^{\prime}$ cannot induce a complete subgraph of $\Gamma$, so $x_{2}=x_{3}=\cdots=x_{n}=1$. Thus, $\operatorname{cent}(x)=p^{-1}\left(G \times\left\langle x_{1}\right\rangle\right) p$, which is clearly free Abelian. Finally, cent $\left(F_{\Gamma}^{\prime} ; x\right)=F_{\Gamma}^{\prime} \cap \operatorname{cent}(x)=p^{-1}\left(\left\langle x_{1}\right\rangle \times G^{\prime}\right) p=p^{-1}\left\langle x_{1}\right\rangle p$, since $G$ is Abelian, so cent $\left(F_{\Gamma}^{\prime} ; x\right)$ is cyclic.

Theorem 3. If the finite graph $\Gamma$ contains no full squares, then $F_{\Gamma}^{\prime}$ is a graph group if and only if it is free.

Proof. If the graph $\Sigma$ has an edge joining $v$ and $w$, then $\operatorname{cent}(v)$ is not cyclic. Thus, if the centralizer of every element of $F_{\Sigma}$ is cyclic, $\Sigma$ must be discrete, which is to say $F_{\Sigma}$ must be free.

Theorems 2 and 3 immediately imply

Corollary 1. If $\Gamma$ contains no full squares, but it does contain a full $n$-gon for some $n>4$, then $F_{\Gamma}^{\prime}$ is not a graph group.

\section{ACKNOWLEDGMENT}

The authors wish to express their thanks to Jacques Lewin for suggesting many of these questions.

\section{REFERENCES}

1. K. Appel and P. Schupp, Artin groups and infinite Coxeter groups, Invent. Math. 72 (1983), 201-220.

2. P. Cartier and D. Foata, Problemes combinatoires de commutation et de rearrangements, Lecture Notes in Math., Vol. 85, Springer-Verlag, Berlin and New York, 1969.

3. W. Dicks, An exact sequence for rings of polynomials in partly commuting indeterminates, J. Pure Appl. Algebra 22 (1981), 215-228.

4. C. Droms, Subgroups of graph groups, J. Algebra 110 (1987), 519-522.

5. K. H. Kim and F. Roush, Homology of certain algebras defined by graphs, J. Pure Appl. Algebra 17 (1980), 179-186.

6. K. H. Kim, L. Makar-Limanov, J. Neggers, and F. Roush, Graph algebras, J. Algebra 64 (1980), 46-51. 
7. S. J. Pride, On Tits' conjecture and other questions concerning Artin and generalized Artin groups, Invent. Math. 86 (1986), 347-356.

8. H. Servatius, Automorphisms of graph groups, J. Algebra (to appear).

Department of Mathematics, College of the Holy Cross, Worcester, Massachusetts 01610

Department of Mathematics, James Madison University, Harrisonburg, Virginia 22807

Department of Mathematics, Worcester Polytechnic Institute, Worcester, Massachusetts 01609 